\title{
Psoriatic Juvenile Idiopathic Arthritis
}

National Cancer Institute

\section{Source}

National Cancer Institute. Psoriatic Juvenile Idiopathic Arthritis. NCI Thesaurus. Code C119044.

A category of juvenile idiopathic arthritis defined by the presence of arthritis with a personal or family history of psoriasis, and features such as dactylitis and nail dystrophy. 